\title{
Philosophiques
}

\section{Psychologie de la " conscience " et science du " behavior "}

\section{J. Nicolas Kaufmann}

Volume 4, numéro 2, octobre 1977

Philosophie et psychologie

URI : https://id.erudit.org/iderudit/203082ar

DOI : https://doi.org/10.7202/203082ar

Aller au sommaire du numéro

Éditeur(s)

Société de philosophie du Québec

ISSN

0316-2923 (imprimé)

1492-1391 (numérique)

Découvrir la revue

Citer cet article

Kaufmann, J. N. (1977). Psychologie de la " conscience " et science du

" behavior ». Philosophiques, 4(2), 313-326. https://doi.org/10.7202/203082ar d'utilisation que vous pouvez consulter en ligne.

https://apropos.erudit.org/fr/usagers/politique-dutilisation/ 


\title{
PSYCHOLOGIE DE LA "CONSCIENCE " ET SCIENCE DU " BEHAVIOR "
}

\author{
par J. Nicolas Kaufmann
}

Il est un fait remarquable dans l'histoire de la psychologie, que durant ses cent ans d'existence, l'objet de cette discipline ait dû changer trois fois de nom. La psychologie débutante de Wundt s'était engagée dans l'étude scientifique de la "conscience". Peu de temps après, avec Watson, Lashley et d'autres, c'est du «bebavior" que la psychologie en tant que science empirique devait traiter. Et finalement, pour Eysenck et les factoristes, le concept clé de la psychologie est le concept de "personnalité".

Ce fait dénote une problématique qui hante la psychologie depuis le moment où elle s'est constituée en science empirique. Elle paraît être une problématique conceptuelle centrale, non seulement du point de vue de l'histoire et de la philosophie des sciences psy. chologiques, mais aussi pour la psychologie en tant que science elle-même. Mes quelques remarques concernant cette problématique porteront sur les deux premiers termes, c'est-à-dire sur le rapport entre une psychologie de la " conscience " et la science du " behavior ". Ces deux " extrêmes " ne permettent pas de caractériser les grandes conceptions et les pratiques thérapeutiques contemporaines qui, pour des raisons qui sous-tendent notre débat, ne se disent ni "psychologie " ni " science", mais analyse : la Psy. choanalyse de Freud, la Daseinsanalyse de Binswanger et la Schicksalsanalyse de Szondi. La démarche analytique que les grands de la psychothérapie ont préconisée ne suit pas le canon de la science, mais s'oriente davantage dans la ligne de l'analyse du langage, de l'herméneutique heideggerienne ou de la "biologie spéculative ".

Le problème du rapport entre la psychologie de la "conscience " et la science du "behavior ", ainsi que celui du statut respectif des deux approches, constitue un problème philosophique. 
Skinner ne s'est pas trompé quand il affirmait : "Le behaviorisme n'est pas l'étude scientifique du comportement, mais une philosophie de la science concernant la matière et les méthodes de la psychologie ${ }^{1}$." Cette philosophie a deux aspects. Nous trouverons chez Skinner, d'une part une préoccupation épistémologique concernant la forme ou le schéma scientifique de l'étude expérimentale du comportement, d'autre part une préoccupation idéologique ou politique préconisant une technologie du comportement et un redressement de la culture par des techniques comportementales. Je m'en tiendrai ici au premier aspect et je ne toucherai le second qu'en autant qu'il présuppose des considérations épistémologiques ${ }^{2}$.

Tout au long de son histoire, de Watson aux postskinnériens, le behaviorisme s'est défini par rapport à la problématique de la conscience et des "mental events", plus exactement contre la psychologie de la "conscience ", en prétendant expliquer le comportement sans référence à des "inner states" ou à des "événements mentaux". En effet, la préoccupation de Watson ne fut-elle pas " to bury subjective subject matters ", sans réussir pour autant à faire disparaître une fois pour toutes ce "stubborn fact of consciousness $n^{3}$, de liquider ce fait récalcitrant de la conscience. Il faut bien admettre que sans la problématique de la conscience il n'y aurait pas eu de psychologie, ni de behaviorisme.

Je voudrais soulever deux séries de questions : a) Comment dans sa conception du comportement, le behaviorisme réussit-il à

1. Skinner, B.F., 1969, Contingencies of Reinforcement : A Theoretical Analysis, New York, Appleton-Century-Crofts. Trad. fr. par M. Richelle, 1971, L'analyse expérimentale du com. portement : Un essai théorique, Bruxelles, Dessart, 291. Skinner a défendu cette position déjà en 1964, "Behaviorism at Fifty", dans : Wann, T.W., (ed.) 1964, Behaviorism and Phenomenology, Contrasting Basis for Modern Psychology, Chicago / London, The University of Chicago Press, 79. Skinner réitère cette même idée dans sa dernière publication, 1974, About Bebaviorism, New York, Knopf, 3.

2. La dimension idéologique et morale est scrutée en profondeur par Mishler, E.G., 1976, "Skinnerism : Materialism Minus Dialectic", dans : The Journal for the Theory of Social Bebavior 6 (2). En suivant Habermas, Mishler tente d'articuler l'idéologie sociale qui soustend la technique expérimentale et la pratique professionnelle du behaviorisme. Le behaviorisme traiterait l'interaction entre acteurs qui, selon Habermas, est fondamentalement communication, en interaction instrumentale où l'un des acteurs est sujet et l'autre objet. Des aspects semblables ont été soulignés par Rothenstreich, N., 1973, "Skinner and Freedom and Dignity' ", dans : Wheeler, H., (ed) 1973, Beyond the Punitive Society, Operant Conditionning: Social and Political Aspects, San Francisco, W.H. Freeman and Company, 217 230 .

3. SKINNER, B.F., 1964, "Behaviorism at Fifty ", op. cit., 84. Skinner n'a jamais changé d'idées à ce sujet. Cf. 1974, About Bebaviorism, op. cit., 69. 
se passer des " mental events $»^{4}$ ? b) La deuxième série de questions portera sur l'analyse du concept clé du béhaviorisme de Skinner, qu'est le concept de "renforcement", en vue d'examiner son pouvoir explicatif.

1. Pourquoi le behaviorisme devait-il finir avec la référence à la conscience, à l'intentionnalité comme sa propriété, à des événements mentaux que sont censés " décrire " les propositions comme "Je me souviens", "Je me décide ", "Je rêve ", "J'ai peur "; etc. ? La réponse est simple : ces propositions relèvent de l'introspection. Déjà W undt' croyait savoir qu'il se trouvait, en tant qu'introspectionniste, être le seul juge de ses "découvertes ", l'introspection étant une affaire strictement privée - ce qui se révélera faux. Wundt ne pouvait pas imaginer comment les descriptions introspectives pussent être soumises à la critique pour satisfaire au critère de la testabilité intersubjective. En effet, comment pouvonsnous vérifier si la description d'un état mental est correcte? se demande le behavioriste. La réponse à cette question étant négative, le behaviorisme devra combattre avec acharnement l'existence des données de la conscience, ou plutôt leur pertinence pour une science.

Or la question de Wundt est une question qui n'a pas de sens, car une observation "introspective " n'est pas plus privée ni moins publique que mon observation de la position de l'aiguille sur un instrument de mesure. Par conséquent, le béhaviorisme s'acharne au départ à se définir contre une problématique qui est mal engagée.

L'interobservabilité qui semble faire défaut dans le cas de la psychologie peut aussi faire problème dans les sciences physiques. En effet, quand Popper discute la procédure de falsification des hy-

4. Il va de soi que l'on mettra ici l'accent sur le "behaviorism "logique ou le "behaviorism " réductionniste, laissant de côté les autres variantes que distinguent soigneusement Koch, S., 1964, "Psychology and Emerging Conceptions of K nowledge as Unitary ", dans : Wann, T.W., (ed.) 1964, Behaviorism and Pbenomenology, op. cit., 1.42, ainsi que Sussman, A.N. 1975, "Mental Entities as Theoretical Entities", dans : American Philosopbical Quarterly $12(4), 277.288$.

5. WUNDT, W., 1874, Grundzüge der physiologischen Psychologie, Leipzig, Engelmann; 1896, Grundriss der Psychologie, Leipzig, Engelmann. Cf. la discussion de Mischel, Th., 1971, "Wundt and the Conceptual Foundations of Psychology ", dans : Pbilosopby and Phenomenological Research 31, 1.26. 
pothèses scientifiques dans ses aspects logiques et opérationnels, il remarque que cette procédure ne s'applique pas aux propositions de base (" basic statements") mêmes. Popper est conscient que son critère de démarcation ne comporte pas seulement des aspects syntaxiques et sémantiques, mais aussi une dimension pragmatique ${ }^{6}:$ l'ac- $^{\prime}$ ceptation des propositions de base, c'est-à-dire des propositions d'observation, et leur correctitude reposent sur un consensus factuel qui ne s'établit que par des interdépendances sociales et communicatives complexes. Leur acceptation repose sur des décisions (conscientes, calculées, inconscientes) qui sont fonction de standards qui ne sont ni empiriques ni analytiques. C'est donc au niveau pragmatique qu'il faudrait finalement chercher les raisons de l'exclusion des propositions introspectives par le behaviorisme. Par ailleurs il faudrait tout d'abord s'enquérir sur le sens pragmatique des propositions introspectives. S'agit-il, dans le cas de la phrase "J'ai la nausée ", d'une proposition descriptive au sens propre du terme ou d'une déclaration? Cette distinction pourrait jeter une nouvelle lumière sur le problème. $\grave{A}$ tout le moins clarifierait-elle les règles méthodologiques à suivre pour l'acceptation de ces propositions (cf. infra).

2. L'exclusion des "mental events" du champ d'investigation de la science du comportement peut prendre différentes formes. Il y a des béhavioristes qui nient purement et simplement l'existence des données de la conscience. Ce fut la seconde position de Watson, J.B.' D'autres estiment que le langage de la conscience doit être réduit à un vocabulaire purement physicaliste. Ce fut la position de Lashley, K.S. ${ }^{8}$, qui défendit le réductionnisme bien avant que Carnap ne formulât ce programme dans les termes du positivisme logique?.

Par delà les différences, l'on peut reconnaître aux diverses formes de behaviorisme un trait commun, à savoir de n'utiliser que des descriptions en termes physiques (au sens large) référant à

6. Popper discute l'interprétation pragmatique de Lakatos et al. dans : 1974, "Replies to My Critics" ("The Problem of Demarcation ", dans : Schilpp, P.A., (ed.), 1974, The Pbilosophy of Karl Popper, La Salle, Illinois, Open Court, 961-1013, notamment 999 ss.

7. 1919, Psychology from the Standpoint of a Bebaviorist, Philadelphia, Lippincott.

8. 1923, "The Behavioristic Interpretation of Counsciousness, I " dans : Psychological Review $30,237.272 ;$ II, 329.353

9. CARnaP, R., 1932-33, “Psychologie in physikalischer Sprache ", dans: Erkenntnis 3, reproduit dans: Ayer, A.J., 1959, Logical Positivism, Glencoe, The Free Press, 165-199. 
des "physical events", par opposition à une psychologie connue sous les titres divers de " psychologie humaniste ", de " psychologie phénoménologique ", approches qui présentent des descriptions et des explications de la conduite humaine en termes d' "intention", d'" attention", de "décision", etc., qui désignent des supposés "événements mentaux ". Ces deux voies de la psychologie ont déjà été thématisées par Brentano, F. ${ }^{10}$ Cette opposition entre deux sortes de psychologie suppose une distinction entre deux sortes d'événements et corrélativement entre deux sortes de descriptions.

Ou bien on prétend que les descriptions en termes d'événements mentaux sont sans référence, qu'elles ne décrivent rien, puisque les événements mentaux sont de pures fictions. Je ne réfuterai pas ici cette thèse que Feigl, $\mathrm{H}^{1{ }^{11}}$ tient, avec force arguments, pour absurdement fausse ("absurdly false"). Mentionnons que l'argument central de Feigl s'appuie sur la différence entre les "hypothèses interprétatives de référence " et les " hypothèses interprétatives d'évidence ". Si l'on utilise dans une théorie psychologique des termes comme "intention ", "intelligence ", etc., ceux-ci ne constituent pas des termes d'observation simples l'expérimentateur ne pouvant pas observer sur autrui des "états mentaux " - mais des termes théoriques que l'on fait par hypothèses (HIR) correspondre à des entités objectives dont l'existence est indirectement attestée par les indicateurs en tant que base d'évidence que l'on associe hypothétiquement (HIE) à ces termes ${ }^{12}$.

Ou bien on admet que les deux descriptions se rapportent à des événements, que les deux sortes de langage ont une référence. Dans ce cas deux possibilités peuvent se présenter : soit que les deux événements sont de nature différente étant donné que leurs descriptions le sont, soit qu'un événement mental est identique à un événement physique, que les deux types d'événements sont identiques en dépit des différences de leurs descriptions. (Cette dernière situation ressemble à la découverte que "l'étoile du matin" est "l'étoile du soir ", l'identité étant énoncée dans une phrase où les

10. 1974, Psychologie vom empirischen Standpunkt, i., Leipzig, Kraus, 1924/25, Livre II, chap. 1, s 5, 125 ss.

11. 1957, "The 'Mental' and the 'Physical" dans: Minnesota Studies in the Pbilosopby of Science II, Concepts, Theories, and The Mind-Body Problem, Minneapolis, University of Minnesota Press, 429.

12. Pour ces deux hypothèses d'interprétation cf. BungE, M., 1969, Scientific Researcb I, The Search for System, Berlin / Heidelberg / New York, Springer, 493-506. 
deux expressions ont la même dénotation, mais pas la même connotation). Cependant, dire que deux types d'événements sont identiques soulève l'épineuse question : à quelles conditions pouvonsnous dire que deux événements sont différents et à quelles conditions qu'ils sont identiques? Une réponse simple serait de prétendre que deux événements sont toujours différents et qu'ils ne sont jamais identiques. Mais Davidson, D. ${ }^{13}$ a montré que la question est beaucoup plus complexe et exige une analyse rigoureuse qui permettrait de traiter plus adéquatement le problème du behaviorisme et le statut des "entités mentales".

3. Pour Skinner, le représentant sans doute le mieux connu du (néo-néo-) behaviorisme, il n'est pas question de faire du comportement une fonction de variables intérieures, qu'elles soient des états mentaux ou autres. Le comportement est une fonction des facteurs de l'environnement entièrement descriptibles en termes physiques. "C'est dans la nature de l'analyse expérimentale du comportement humain de se détourner des facteurs autrefois assignés à l'homme autonome et de les transférer un par un aux va. riables de l'environnement qui exercent le contrôle ${ }^{14}$. "Nous trouvons dans ce " credo " partiellement la variante méthodologique du behaviorisme et surtout le programme réductionniste dont j'interrogerai quelques prémisses.

“Transférer les facteurs un à un à des variables de l'environnement ", ce n'est rien dire à propos du comportement, mais c'est prophétiser dans quelle direction la science du comportement devra nécessairement progresser. On peut bien cröire que la science démontrera un jour que le comportement est entièrement déterminé par des facteurs de l'environnement ; mais il reste à se demander quelle serait la base scientifique pour un tel pronostic. Que la science de la nature ne peut pas s'intéresser à d'autres facteurs qui passeraient pour des fictions, telle la " vis dormitiva ${ }^{19}$, cela n'im-

13. 1971, "The Individuation of Events", dans: Rescher, N., et al. (eds.), 1971, Essays in Honor of Carl G. Hempel, Dordrecht, Reidel, 216-234; 1970, "Mental Events ", dans : Fact and Experience, Swanson and Forster (ed.), Amherst 1970; 1974, "Psychology as Philosophy ", dans : Brown, S.C., (ed.), 1974, Pbilosopby of Psychology, London, Macmillan, 41-53. Des questions semblables sont abordées pat Block, W.J., Fodor, J.A., 1972, "What Psychological States are not ", dans : Pbilosopbical Research 81 (2), 159.181.

14. SKInNER, F.B., 1971, Beyond Freedom and Dignity, New York, Knopf, 195.

15. SKINNER, F.B., 1971, Beyond Freedom and Dignity, op. cit., 8. 
plique strictement rien pour une science du comportement rétorquerait Chomsky, N. ${ }^{16}$.

Considérons deux descriptions d'une de mes actions. "Je lève mon bras "; un observateur dirait : "Le bras se lève ". Mais en termes de physique, la dernière description concerne un mouvement apparent, le mouvement réel étant beaucoup plus complexe du fait que cette salle se trouve transportée en direction Est avec une vitesse de 700 milles/h environ. Pourquoi le behavioriste ne décrit-il pas le mouvement de mon bras en termes de mouvement réel ? Pourquoi ne tient-il pas cette description qui, du point de vue physicaliste, est beaucoup plus complète, pour la description correcte d'un comportement? Cette question oblige à examiner le programme réductionniste qu'endosse un certain behaviorisme. Le postulat méthodologique fondamental est que " toutes les propositions psychologiques, pour avoir un sens, doivent décrire des occurrences et des événements physiques (au sens large) ". Carnap dirait plus clairement que les phrases "Je suis angoissé ", "J'ai soif " n'ont pas de sens dans cette forme, puisqu'elles ne nous renseignent pas sur les conditions qui doivent être réalisées pour les rendre vraies, et que les propositions en question ou les termes qui y figurent ne réfèrent pas à des événements ou à des propriétés observa. bles comme des déplacements, des contractions musculaires, etc. .

D'une part, on pourrait dire en guise de critique qu'adopter cette position c'est confondre les critères de sens avec les critères de vérité. Cette position a été démontrée intenable par Hempel et d'autres". D'autre part, si des phrases comme "J'ai soif ", pour avoir un sens et pour être admissibles dans une psychologie scientifique, doivent être réduites à des phrases comme "Le taux de glucose baisse en deçà du seuil X ", "Tel ou tel processus se produit dans le système nerveux central "..., on peut se demander ce que ferait le physicaliste dans le cas de douleurs ou de soif hallucinées, et comment il distinguerait les hallucinations vraies des hallucinations fausses. Personne pourtant ne nie qu'il s'agisse là de

16. 1973, "The Case against Skinner ", dans : Matson, F.W., (ed.) 1973, Witbout/Witbin, Behaviorism and Humanism, Belmont, Brooks-Cole, 62, 63.

17. Hempel, C.G., 1965, "Empiricist Criteria of Cognitive Significance: Problems and Changes ", dans : Hempel, C.G., 1965, Aspects of Scientific Explanation, New York, The Free Press, 1968, 101-133 ; LADRIÈRE, J., 1970, “ La tentative néo-positiviste ", dans : Ladrière, J., 1970, L'articulation du sens, Paris, Aubier/Montagne, 73-91. 
phénomènes proprement psychologiques qui semblent résister à la tentative réductionniste.

On aura remarqué que ce qui est en cause, c'est la procédure de réduction d'un langage en première personne à un langage en troisième personne, et qu'il y va en fin de compte de l'élimination du langage en première personne où figurent des termes qui réfèrent à des états mentaux. Or une très grande partie des recherches en psychologie ne peut pas se passer du langage en première personne. Bien des travaux des behavioristes mêmes sont tributaires des résultats des gestaltistes qui, eux, ne pratiquaient nullement cette réduction, mais admettaient des propositions de protocole par auto-observation. Zenner, $\mathrm{R} .{ }^{18}$ remarque que " in objective experiments with rats Lashley has demonstrated the major facts concerning perceptual grouping, figure-ground differentiation and the like, originally reported by Wertheimer, Rubin and Köhler in human "introspective " experiments. The implication that human reports on experience were therefore dispensable is incorrect". Nombreux seraient les exemples qui démontreraient la fécondité du matériel "introspectif " précisément dans des recherches qui se font d'un point de vue strictement behavioriste.

Le langage en première personne fait certainement problème du point de vue de la méthode scientifique. Les phrases "J'ai soif ", "J'ai peur ", " Je vois l'image à l'envers " . . et les propositions de protocole "Le taux de glucose descend au deçà du seuil $X$ ", "La dose d'adrénaline dans le sang est $Y$ » . . diffèrent dans leur sens non seulement du point de vue sémantique, mais surtout du point de vue de la situation pragmatique. Examinons la différence.

1) "J'ai peur".

2) “La dose d'adrénaline dans le sang est $Y$ ".

La phrase 1) appartient manifestement à un langage autoimplicatif où le locuteur (le sujet dont le comportement est en cause) accomplit un acte illocutoire, dans la terminologie de Searle, qui est bien différent de l'acte de celui qui prononce la phrase 2). La situation pragmatique est la suivante:

18. 1957, "Experience of the Individual", dans : Minnesota Studies in the Philosophy of Science, op. cit., 363. 
1) S dit: "J'ai peur".

2) O dit: "La dose d'adrénaline dans le sang est y ".

Le locuteur $\mathrm{O}$ dans le cas 2) produit un acte illocutoire que Searle, J.R. ${ }^{19}$ classerait dans les "representatives"; le locuteur $\mathrm{O}$ se commet pour la vérité ou la fausseté du contenu propositionnel de la phrase, et cet acte n'est pas identique à celui de $\mathrm{S}$ dont on analyse le comportement. Par contre, dans le cas 1), l'acte illocutoire fait partie du comportement même du sujet en cause, le comportement "d'avoir peur"; l'expression ne consiste pas à relater un événement qui pourrait être le cas ou non, comme dans 2), mais à révéler la manière de $S$ d'appréhender une situation. Dans 1), contrairement à 2), il s'agit d'un usage expressif du langage. Ce qui caractérise aux yeux de Searle ce type d'usage du langage, c'est qu'il soit impossible de stipuler pour de telles expressions les conditions de succès ou de "raté ". Le programme réductionniste sousentend que le psychologue scientifique doit faire un choix entre les deux usages (" expressif " et "représentatif") du langage. Le réductionniste déclare implicitement que le premier registre est "irrelevant " pour l'étude du comportement. Mais cette conclusion ne découle pas du statut des deux registres du langage et de la situation pragmatique. Elle procède d'un choix que le behavioriste fait sur des bases qu'il devra expliciter et justifier. Il y en a qui pensent que ce choix du behaviorisme radical constitue non un gain, mais une perte, un appauvrissement des notions psychologiques du langage ordinaire ${ }^{20}$.

Dans la perspective de Skinner, il n'est pas nécessaire de réduire des énoncés, comme "J'ai soif ", pour les rendre significatifs, à la physiologie. Skinner dit très clairement que faire cela, c'est abandonner la psychologie ${ }^{21}$. Mais les énoncés en question doivent être considérés comme "verbal behavior " pour lequel on peut chercher les variables qui contrôlent cette sorte de comportement. Ces variables se trouvent dans l'environnement. Le comportement verbal, comme tout autre comportement, est une fonction de (E.R.). Analysons cette procédure où un énoncé en première personne est traité comme un "behavior".

19. "A classification of Illocutionary Acts", dans : Gunderson (ed.), Minnesota Studies in the Philosopby of Science VI (à paraître) 354 ss.

20. Cf. Bloomfield, T.M., 1976, "About Skinner : Notes on the Theory and Practice of 'Radical Behaviorism' ", dans: Phil. Soc. Sci. 6, 75-82.

21. SKInNER, B.F., 1971, Beyond Freedom and Dignity, op. cit., 3, 31 . 
Skinner a lui-même consacré une étude au comportement verbal $^{22}$. Ses études sur le comportement verbal ont pour but de montrer que les phrases du genre de celles mentionnées sont le résultat de variables de l'environnement et d'un renforcement de l'opérant en tant qu'augmentation de la probabilité de la réponse dans des circonstances données.

Je rappelle ici la critique que Chomsky a faite de l'" explication" skinnérienne du comportement verbal ${ }^{23}$. Chomsky demande comment on peut expliquer, à partir d'un répertoire de phrases déterminé, - répertoire formé d'expressions en nombre fini qui se trouvent effectivement renforcées par l'usage dans le passé (selon Skinner), - que la probabilité de produire une nouvelle phrase française, qui n'appartient pas par définition au répertoire, est plus grande que la probabilité de produire une phrase chinoise? Skinner devrait répondre à la question de Chomsky que c'est la similitude de la nouvelle phrase avec l'ancienne qui rend cette occurrence plus probable. Mais juger de la similitude, rétorque Chomsky, c'est mettre en jeu des critères d'ordre grammatical ou autre, qui sont forcément des schèmes abstraits, des règles qui ont un statut abstrait, des " inner states " dont Skinner ne sait que faire, mais qu'il doit présupposer pour rendre sa "théorie " plausible. Cela signifie que l'on chasse les états mentaux par la grande porte pour les réadmettre par la porte de service. Bien des néo-behavioristes qui se sont attaqués avec rigueur au problème de l'explication du comportement d'apprentissage, dont le comportement verbal, se sont rendu compte qu'une telle théorie ne peut pas se passer des postulats relatifs à des "inner states $»^{24}$.

\section{II}

Examinons brièvement le concept de "renforcement". Si nous demandons ce qu'il faut entendre par " renforcement " - il

22. Skinner, B.F., 1957, Verbal Bebavior, New York, Appleton-Century-Crofts. Il a également consacré des chapitres ou des pages dans: 1969, Contingencies of Reinforcement, op. cit., 1974, About Bebaviorism, op. cit., 88.102.

23. ChOmsky. N., 1973, "The Case Against Skinner ", op. cit., et 1959, "Review of Verbal Behavior by B.F., Skinner ", dans : Language 35, 26.58.

24. Cf. Nelson, R.J., 1969, "Behaviorism is False ", dans : Journal of Philosophy 66, 417.453 ; Nelson, R.J., 1975, " Behaviorism, Finite Automata, and Stimulus Response Theory ", dans : Theory and Decision 6 (3), 249-267 ; Boneau, C.A., 1974, "Paradigm Regained? Cognitive Behaviorism Refuted", dans: American Psychologist 29 (5), 297-310. 
s'agit du concept et non des techniques de laboratoire -, nous nous heurtons à quatre difficultés. J'espère montrer par cette analyse que l'entreprise skinnérienne n'est pas une entreprise scientifique.

1. Le terme de "renforcement", (positif ou négatif), étant défini par l'augmentation (la diminution) de la probabilité de la réponse à une stimulation, le renforcement est une mesure de probabilité. Dans ce cas, le concept paraît avoir une signification plutôt métathéorique. Le concept ainsi défini ne renseigne pas sur le comportement, mais sur la manière dont se "comportent " des variables. Skinner n'aurait certainement pas accepté un tel point de vue, car, pour lui, le renforcement est " quelque chose".

2. Si le renforcement est "quelque chose ", il pourrait luimême être de l'ordre du comportement, disons du comportement perceptif. Ce point de vue aussi fut vigoureusement combattu par des skinnériens - et pour cause. Locke ${ }^{25}$ qui a étudié la question a mis le doigt sur l'inexactitude des travaux de Greenspoon, J. ${ }^{26}$ qui prétendait démontrer que le renforcement ne relevait pas de la perception, de l'« awareness " que le sujet pourrait avoir durant la séquence de conditionnement. Locke mentionne une série de travaux, expressément conçus pour tester l'hypothèse de Greenspoon ; ces travaux prouvent exactement le contraire : les sujets qui se trouvaient réellement renforcés étaient justement ceux qui se sont rendu compte que le " $\mathrm{Hm}, \mathrm{hm}$ ! " de Greenspoon était associé aux mots au pluriel, ce qui augmentait la probabilité de réponse, c'est-à-dire la probabilité de produire des mots au piuriel ; ils développaient des stratégies en fonction de ce qu'ils ont perçu' ${ }^{27}$.

25. Locke, E.A., 1972, "Critical Analysis of the Concept of Causality in Behavioristic Psychology", dans : Psychological Reports, 31, 175-197.

26. GReEnspoon, J., 1955, "The Reinforcing Effect of Two Spoken Sounds on the Frequency of Two Responses ", dans : American Journal of Psychology 68, 409-16.

27. Pour GRINGs, W.W., 1973, "The Role of Counciousness and Cognitive on in Autonomic Behavior Change ", dans : McGuigan, F.J., Schoonover, R., (eds), 1973, The Psychology of Thinking, New York, Academic Press, Bandura, A., 1969, Principles of Bebavior Modification, New York, Holt, Rinehart \& Winston, Dulanay, D.E., 1968, "Awareness, Rules and Propositional Control : A Confrontation with S-R-Behavior Theory ", dans: Dixon, T.R., Horton, D.L., (eds), 1968, Verbal Bebavior and General Bebavior Theory, Englewood Cliffs, N.J., Prentice-Hall, un renforcement sans conscience (" awareness ") semble également fort discutable dans le cas du conditionnement du comportement humain. 
Le comportement étant selon la formule de Skinner fonction de $\mathrm{E}$ et de $\mathrm{R}$, le renforcement lui-même relève maintenant d'un autre type de comportement (la perception) ; ce comportement est à son tour fonction de $\mathrm{E}$ et de $\mathrm{R}$, et ainsi de suite. Skinner est obligé de s'engager dans une régression à l'infini.

3. Pour notre étonnement, Skinner suggère lui-même cette régression. N'a-t-il pas inlassablement répété, dans son plaidoyer pour une technologie du comportement ${ }^{28}$, que, pour modifier le comportement de quelqu'un, il suffisait de changer simplement certaines conditions de l'environnement, qu'au lieu de punir, il fallait conditionner? Ce qu'il faut donc modifier dans l'environnement, c'est le comportement punitif des autres. Par conséquent, l'on modifie le comportement en modifiant le comportement, comme ce qui renforce le comportement verbal, c'est le comportement verbal. C'est à cette trivialité qu'aboutit Skinner une fois qu'il quitte le laboratoire.

4. Dire que le renforcement augmente la probabilité de la réponse n'explique strictement rien, car ce qu'il faudrait savoir, c'est pourquoi la probabilité augmente. Si jamais Skinner répondait que c'est à cause du renforcement, je lui ferais remarquer que c'est ainsi qu'il a défini le concept de "renforcement". Dans ce cas, nous aurons une tautologie et non un principe empirique. Plus exactement, comme le signale Bloomfield, T.M. ${ }^{29}$ le principe de renforcement n'est pas, contrairement à la "loi de l'effet" de Thorndike ou de Hall, un postulat théorique que l'on mettrait expérimentalement au test. C'est un principe d'action indiquant ce qu'il convient de faire dans des circonstances déterminées en vue d'atteindre un objectif déterminé. Il prend la forme d'un impératif technique.

Ce qui paraît extrêmement critiquable, c'est que Skinner prétend faire de la science tout en avouant sans ambages que sa propre théorie n'explique rien. "Je ne suis pas intéressé d'expliquer pourquoi quelque chose est renforçant ... Je ne peux pas expliquer pourquoi la nourriture est renforçante pour un animal affamé .... $\cdots^{30}$. Une théorie qui n'explique rien n'est pas une théorie scientifique. En cela Skinner est loin des travaux de Pavlov,

28. SKINNER. B.F., 1971, Beyond Freedom and Dignity, op. cit., 259.

29. BloOMField. T.M., 1976, "About Skinner ", op. cit., 76.

30. SkinNer, B.F., 1964, "Behaviorism at Fifty ", op. cit., 104. 
le père de la théorie du conditionnement classique. En effet, celuici nous fournit une théorie, quoique contestable et contestée, qui est en mesure d'expliquer quelqus perı ce qui s'observait en laboratoire. Par contre, le contenu théorique de l'oeuvre de Skinner est fort maigre. Ses écrits se trouvent remplis d'innombrables descriptions des dispositifs expérimentaux, des multiples procédures de conditionnement qui varient en fonction du " matériel " (humain ou animal) sur lequel il travaille, d'actogrammes, de statistiques . . Mais on n'y trouve pas d'axiomes et pas de théorèmes. Pour les langues méchantes, "Skinner's arbitrary identification of science with the procedures of operant conditionning, his unsupported and dogmatic rejection of the notion of human responsability and, hence, of human agency, his extravagant testimonials to a 'behavioral technology', which is, I am confident, still no more than a future threat - all this mélange of amateurish metaphysics, self-advertising 'technology', and illiberal social policy adds up to a document that is disservice to scientists, technologists, and to all who are seriously trying to improve the human condition $n^{31}$.

Dès lors, la prétention de Skinner ${ }^{32}$ que l'investigation du conditionnement de l'opérant "remplace progressivement la fonction explicative autrefois assignée à des états mentaux, projets et intentions " est parfaitement vide puisque le concept clé de la théorie skinnérienne, le concept de "renforcement ", n'a pas de pouvoir explicatif. Skinner est un moraliste déguisé ; le skinnérianisme est une technologie, et c'est aux techniciens du conditionnement, dont les succès ne sont nullement contestés ici, qu'il convient, en guise de conclusion, de poser quelques questions.

Dans l'optique du conditionnement de l'opérant, est-ce que tout comportement est conditionnable ou seulement une classe de comportements déterminée? On peut par exemple se demander s'il est possible, par des techniques appropriées, de conditionner un comportement pacifique qui reste définitif. Peut-on tracer une ligne de démarcation entre les comportements conditionnables et ceux qui ne le seraient pas? Possède-t-on des critères autres que l'essai et l'erreur pour ce faire?

31. BlACK. M., 1973, "Some Aversive Responses to a Would-be Reinforcer ", dans : Wheeler, H., (ed.), 1973, Beyond the Punitive Society, op. cit., 133-34.

32. 1971 , Beyond Freedom and Dignity, op. cit., 18. 
Il est évident que pour qu'un organisme soit conditionnable, celui-ci doit se trouver dans un état initial approprié, avant que l'on applique une séquence de conditionnement. Quelqu'un qui dort ou se trouve dans le coma est manifestement dans un état inapproprié pour pratiquement toutes les techniques de conditionnement. Le problème méthodologique et pratique est le suivant : Comment le technicien du comportement peut-il savoir que ses clients, surtout quand il s'agit d'un " matériel " humain, se trouvent dans un état initial approprié, qu'ils sont conditionnables par une méthode déterminée? Et s'il sait qu'ils ne le sont pas, comment peut-il les mettre dans un état initial approprié? Par quelle technologie établirait-il ces états? Si jamais de telles techniques pouvaient être "inventées" seraient-elles plus raffinées que la simple incarcération ou la privation, moyens qui se sont révélés très efficaces dans le cas des rats et des pigeons?

Département de Philosophie, Université du Québec à Trois-Rivières. 